\title{
A Benchmark Library and a Comparison of Heuristic Methods for the Linear Ordering Problem
}

\author{
Rafael Martí • Gerhard Reinelt • \\ Abraham Duarte
}

November 18, 2010

\begin{abstract}
The linear ordering problem consists of finding an acyclic tournament in a complete weighted digraph of maximum weight. It is one of the classical NP-hard combinatorial optimization problems. This paper surveys a collection of heuristics and metaheuristic algorithms for finding near-optimal solutions and reports about extensive computational experiments with them. We also present the new benchmark library LOLIB which includes all instances previously used for this problem as well as new ones.
\end{abstract}

\section{Introduction}

Let $D_{n}=\left(V_{n}, A_{n}\right)$ denote the complete digraph on $n$ nodes, where $V_{n}$ is the set of nodes and $A_{n}$ the set of arcs. A tournament $T$ in $A_{n}$ consists of a subset of arcs containing for every pair of nodes $i$ and $j$ either $\operatorname{arc}(i, j)$ or $\operatorname{arc}(j, i)$, but not both. $T$ is an acyclic tournament if it does not contain any directed cycle. Obviously, an acyclic tournament induces an ordering $\left\langle v_{i_{1}}, v_{i_{2}} \ldots, v_{i_{n}}\right\rangle$ of the nodes (and vice versa): node $v_{i_{1}}$ is the one with no entering $\operatorname{arcs}$ in $T, v_{i_{2}}$ has exactly one entering arc, etc., and $v_{i_{n}}$ is the node with no outgoing arc. Given arc weights $w_{i j}$ for every pair $i, j \in V_{n}$, the linear ordering problem (LOP) consists of finding an acyclic tournament $T$ in $A_{n}$ such that $\sum_{(i, j) \in T} w_{i j}$ is maximal, or in other words, of finding an ordering of the nodes such that the sum of the weights of the arcs compatible with this ordering is maximal.

R. Martí

Dept. de Estadística e Investigación Operativa, University of Valencia, Valencia, Spain E-mail: rafael.marti@uv.es

G. Reinelt

Institute for Computer Science, University of Heidelberg, Heidelberg, Germany

E-mail: gerhard.reinelt@informatik.uni-heidelberg.de

A. Duarte

Dept. Ciencias de la Computación, University Rey Juan Carlos, Madrid, Spain

E-mail: abraham.duarte@rjc.es 
Alternatively, the problem can be defined as a matrix problem. Given an $(n, n)$ matrix $C=\left(c_{i j}\right)$ the triangulation problem is to determine a simultaneous permutation of the rows and columns of $C$ such that the sum of superdiagonal entries becomes as large as possible (or equivalently, the sum of subdiagonal entries is as small as possible). Note, that it does not matter if diagonal entries are taken into account or not. Obviously, by setting arc weights $w_{i j}=c_{i j}$ for the complete digraph $D_{n}$, the triangulation problem for $C$ can be solved as linear ordering problem in $D_{n}$. Conversely, a linear ordering problem for $D_{n}$ can be transformed to a triangulation problem for an $(n, n)$-matrix $C$ by setting $c_{i j}=w_{i j}$ and the diagonal entries $c_{i i}=0$ (or to arbitrary values).

The LOP can also be seen as a problem of ranking objects. Suppose there are $n$ objects which are to be ranked where there is a benefit $c_{i j}$ if object $i$ is ranked before object $j$. The task of finding a linear ranking maximizing the sum of benefits w.r.t. this ranking amounts to solving a LOP or triangulation problem.

In the following we do not distinguish between the graph and the matrix or the ranking problem and denote the objective function coefficients by $c_{i j}$.

Note, that if a constant is added to both entries $c_{i j}$ and $c_{j i}$ or if we take diagonal entries into account, the optimality of an ordering is not affected by this transformation. However, the quality of bounds does change. If we add large constants, then every feasible solution is close to optimal and no real comparison of qualities is possible (which is very relevant when we are comparing heuristics). Therefore we transform every problem matrix $C$ to its normal form satisfying the following conditions:

i. All entries of $C$ are integral and nonnegative,

ii. $c_{i i}=0$, for all $i=1, \ldots, n$,

iii. $\min \left\{c_{i j}, c_{j i}\right\}=0$, for all $1 \leq i<j \leq n$.

Note that this normal form is unique.

The LOP can be formulated as a $0 / 1$ linear integer programming (IP) problem as follows. We use $0 / 1$ variables $x_{i j}$, for $(i, j) \in A_{n}$, stating whether arc $(i, j)$ is present in the tournament or not. Taking into account that a tournament is acyclic if and only if it does not contain any dicycle of length 3 , it is easily seen that the LOP can be formulated as the 0/1-IP

$$
\begin{aligned}
\max \sum_{(i, j) \in A_{n}} c_{i j} x_{i j} & \\
x_{i j}+x_{j i} & =1, \text { for all } i, j \in V_{n}, i<j, \\
x_{i j}+x_{j k}+x_{k i} & \leq 2, \text { for all } i, j, k \in V_{n}, i<j, i<k, j \neq k, \\
x_{i j} & \in\{0,1\}, \text { for all } i, j \in V_{n} .
\end{aligned}
$$

This IP is the basis for approaches to solve the LOP to optimality. If we replace the constraints " $x_{i j} \in\{0,1\}$ " by " $0 \leq x_{i j} \leq 1$ " then we obtain a linear programming problem, whose optimum objective function value provides an upper bound on the optimum value of the LOP. In cases where we do not know optimum solutions to the benchmark problems, we will usually take this 
upper bound for assessing the quality of heuristics. We will not address the computation of optimum solutions in this paper.

The LOP has been the subject of study for a long time. Applications mentioned in the literature are, for instance, aggregation of individual preferences [11], triangulation of input-output tables [26,8], determination of ancestry relationships [15], scheduling with preferences [4], assessment of corruption perception [1] and minimizing crossing numbers in graph drawing [21].

This paper is organized as follows. In Section 2 we describe simple heuristic approaches for the LOP. Section 3 discusses metaheuristics summarizing the most relevant work in approximate optimization for the LOP. The benchmark problems are described in Section 4. In Section 5 we report extensive experimental results targeting both short and medium computation time. Some final remarks and an appendix with the best known solution values conclude the paper.

\section{Heuristics}

In this section we review construction and improvement heuristics. Construction methods obtain a solution, i.e., a linear ordering from scratch adding iteratively one node at each step. Improvement heuristics, also called local search or ascent methods (in the case of maximization problems) start from the solution obtained with a construction method and iteratively improve it by performing local changes usually referred to as moves. The different types of moves and selection strategies characterize the various heuristics. In this section we consider the two typical moves: insertion and exchange.

\subsection{Construction method of Chenery and Watanabe}

The earliest heuristic for the LOP was introduced in 1958 by Chenery and Watanabe [8]. It concerns an application in economy and it is a simple method to rank the sectors of input-output tables. Sectors having a large share of outputs to other sectors should be ranked first. We can view this method as a greedy algorithm in which the attractiveness $a_{i}$ of sector $i$ is the sum of the elements in its corresponding row, i.e.,

$$
a_{i}=\sum_{j=1}^{n} c_{i j}
$$

The method successively constructs an ordering by selecting in each step the most attractive sector among the sectors not ranked so far. 
2.2 Construction methods of Aujac and Masson

Aujac and Masson [2] also rank sectors based on coefficients. The output coefficient $b_{i j}$ of a sector $i$ with respect to another sector $j$ is defined as

$$
b_{i j}=\frac{c_{i j}}{\sum_{k \neq i} c_{i k}} .
$$

The first method (AM-0) intends to rank sector $i$ before sector $j$ whenever $b_{i j}>b_{j i}$. Since this is impossible in general, it heuristically tries to find a linear ordering with few contradictions to this principle. Similarly, the second method (AM-I) defines input coefficients, where $c_{i j}$ is divided by the sum of the entries in the corresponding column, and ranks sectors according to them.

\subsection{Construction methods of Becker}

Becker [3] proposed a construction method (Bci) related to the previous ones in that it calculates special quotients to rank the sectors. For each sector $i$ the number

$$
q_{i}=\frac{\sum_{k \neq i} c_{i k}}{\sum_{k \neq i} c_{k i}}
$$

is computed. The sector with the largest quotient $q_{i}$ is then ranked highest. Its corresponding rows and columns are deleted from the matrix, and the procedure is applied to the remaining sectors.

Becker also proposed a second construction method (Bcr) based on rotations, which seems a local search method. Starting from a random ordering, at each iteration this method tries to improve the current ordering $O=$ $\left\langle i_{1}, i_{2}, \ldots, i_{n}\right\rangle$ by evaluating all orderings $\left\langle i_{m+1}, i_{m+2}, \ldots, i_{n}, 1,2, \ldots, i_{m}\right\rangle$, for $m=1,2, \ldots, n-1$. If the best one among them improves $O$, the method takes it as the new current ordering and continues, otherwise it stops.

\subsection{Construction methods based on insertions}

We have included two further constructive methods, BI1 and BI2, based on insertions. They basically select an arbitrary unassigned object and insert it into the partial solution at the currently best possible position. Let $\left\langle i_{1}, \ldots, i_{k}\right\rangle$ be the current partial ordering. BI1 computes for every object $l$ not ranked so far coefficients

$$
q_{t}=\sum_{j=1}^{t-1} c_{i_{j} l}+\sum_{j=t}^{k} c_{l i_{j}}
$$


for $1 \leq t \leq k$, and inserts $l$ at the position with maximum coefficient. Alternatively, BI2 uses coefficients

$$
q_{t}=\sum_{j=1}^{t-1} c_{i_{j} l}+\sum_{j=t}^{k} c_{l i_{j}}-\sum_{j=1}^{t-1} c_{l i_{j}}-\sum_{j=t}^{k} c_{i_{j} l} .
$$

\subsection{Improvement methods based on insertions}

Removing a node from the current ordering and inserting it at a different position is a simple possibility for searching for an improvement. Laguna et al. [24] define move $\left(O_{j}, i\right)$ as the modification which deletes $O_{j}$ from its current position $j$ in permutation $O$ and inserts it at position $i$ (i.e., between the objects currently in positions $i-1$ and $i$ ). The move value is the difference between the objective function values after and before the move. The method LSi scans the list of nodes (in the order given by the current permutation) in search for the first node whose movement results in an strictly positive move value.

A different approach based on insertions is the method known as $k$-opt, which basically selects $k$ elements of a solution and locally optimizes with respect to these elements (i.e., considers all subsets of $k$ objects $O_{i_{1}}, \ldots, O_{i_{k}}$ in the current permutation and finds the best assignment of these objects to the positions $\left.i_{1}, \ldots, i_{k}\right)$. Since the number of possible new assignments grows exponentially with $k$, we have only implemented 2-opt and 3-opt.

\subsection{Improvement based on exchanges}

This heuristic checks whether the objective function can be improved if the positions of two objects in the current ordering are exchanged. All such possibilities are checked and the method stops when no further improvement is possible this way.

In [24] a limited version of this improvement method is considered. The authors tested a method in which only contiguous sectors are considered for exchange (swap) and concluded that better solutions can be obtained with general insertions. However, as far as we know, general exchanges (between any pair of sectors) have never been tested. We will consider them in our computational comparison.

\subsection{Kernighan and Lin improvement}

Kernighan and Lin [22] introduced compound moves as a series of simple moves. In contrast to pure improvement heuristics, they allow that some of the simple moves (such as insertions or exchanges) are not improving. However, it is required that the composition produces an improvement. Algorithm KL1 considers sequences of up to $n$ exchange moves. Each of the simple moves is 
the best available one, but it can be a non-improving. If the composition of $k$ moves, for some $1 \leq k \leq n$, results in an improvement, then it is performed. Otherwise the moves are discarded and the original solution is restored. Variant KL2 works in the same way composing sequences of insertion moves.

\subsection{Local Enumeration}

This heuristic chooses windows $\left\langle i_{k}, i_{k+1}, \ldots, i_{k+L-1}\right\rangle$ of a given length $L$ of the current ordering $\left\langle i_{1}, i_{2}, \ldots, i_{n}\right\rangle$ and determines the optimum subsequence of the respective objects by enumerating all possible orderings. The window is moved along the complete sequence until no more improvements can be found. We implemented this method, denoted LE, with length $L=8$.

\section{Metaheuristics}

In recent years, a series of methods have appeared under the name metaheuristics, a term coined by Glover [14] in 1986. Basically, we consider a metaheuristic as combination of simple heuristics with some scheme of randomization and additional features, which can be interpreted as learning mechanism and systematic exploration of search spaces. The following approaches fall into this category. We give only short descriptions of these procedures here because they are discussed in depth in the recent book by Martí and Reinelt [27].

\subsection{KLM - Kernighan and Lin multi-start method}

Multi-start procedures exploit a local or neighborhood search procedure by applying it from multiple random initial solutions. It is well known that search methods based on local optimization that aspire to find global optima usually require some type of diversification to overcome local optimality. Without diversification, such methods can reduce to tracing paths that are confined to a small area of the solution space, making it almost impossible to find a global optimum. We apply the Kernighan and Lin method from different initial random solutions until a pre-specified time limit is reached.

\subsection{CK - Chanas and Kobilansky multi-start method}

Chanas and Kobylanski [8] proposed a multi-start method, called CK, based on the following symmetry property. If the permutation $O=\left\langle O_{1}, O_{2}, \ldots, O_{n}\right\rangle$ is an optimum solution to the maximization problem, then an optimum solution to the minimization problem is $O^{*}=\left\langle O_{n}, O_{n-1}, \ldots, O_{1}\right\rangle$. The method utilizes this property to escape local optimality: once a local optimum solution $O$ is found, the process is re-started from the permutation $O^{*}$ (REVERSE operation). 
In a global iteration, CK performs insertions as long as the solution improves. Given a solution, the algorithm explores move $\left(O_{j}, i\right)$ for all $O_{j}$ and $i$ in $O$, and performs the best one. When no further improvement is possible, it generates a new solution by applying the REVERSE operation from the last solution obtained, and performs a new global iteration. The method halts when the best solution found cannot be improved further in the current global iteration.

\subsection{GRASP - Greedy Randomized Adaptive Search Procedure}

Each GRASP iteration [12] consists of constructing a trial solution and then applying an improvement procedure to find a local optimum (i.e., the final solution for that iteration). In [5] we can find several GRASP algorithms embedded in a scatter search procedure for the LOP. The best one combines a randomized adaptive construction based on the greedy evaluation $e$, with a local search based on a best insertion (i.e., move to the best solution in the neighborhood defined by insertion moves). The evaluation $e(i)$ of object $i$ is the sum of the elements in its corresponding matrix row:

$$
e(i)=\sum_{j=1}^{n} c_{i j} .
$$

\subsection{TS - Tabu Search}

Tabu search [16] is a metaheuristic that guides a local search procedure to explore the solution space beyond local optimality by allowing non-improving moves in the local search. The basic tabu search algorithm proposed in [24] implements a short-term memory structure alternating two phases: intensification and diversification. It is based on insertions as the improvement phase of the GRASP algorithm described above. However, instead of scanning the objects in search for a move in their original order, they are randomly selected in the intensification phase based on a measure of influence.

The basic method is complemented with a long-term intensification based on the path relinking methodology [25], and with a long-term diversification based on the REVERSE operation proposed in CK. Both long-term strategies incorporate frequency information (memory structures) recorded during the application of the short-term phase.

\subsection{SS - Scatter Search}

Scatter search is an evolutionary or population based method [25] that operates on a relatively small set of solutions, called reference set, combining them to obtain new and hopefully better solutions. In [5] an SS algorithm is described 
in which solutions are generated with a diversification method, combined with a min-max method based on a voting scheme, and improved with the local search method employed in the GRASP method referenced above.

Some preliminary tests [5] disclose the best strategies to implement these three methods. We can highlight the use of a frequency-based procedure as the diversification generator method and the combination of multiple solutions.

\subsection{VNS - Variable Neighborhood Search}

Variable neighborhood search [20] is based on a simple and effective idea: a systematic change of the neighborhood within a local search algorithm. In [13] a VNS algorithm is proposed based on neighborhoods $\mathcal{N}_{k}, k=1, \ldots, n$, where the neighborhood of the solution $p, \mathcal{N}_{k}(p)$, is the set of solutions that are obtained when we apply $k-1$ insertion moves to $p$.

The VNS algorithm applies three steps: shaking, in which the current solution is perturbed, improving, in which a local optimum with respect to the current neighborhood is obtained, and updating, in which a change in the neighborhood is performed. The authors proposed in [13] a hybrid algorithm in which VNS is combined with memory structures for improved outcomes.

\subsection{GA and MA - Genetic and Memetic Algorithms}

In [31] a genetic algorithm coupled with a local search procedure, called memetic algorithm, is developed. As in SS above, it basically consists of generating, improving, and combining solutions. In the initialization, a population of individuals is obtained by first generating a set of random permutations (solutions) and then applying a local search procedure, based on insertions, to each of them.

In each iteration of the algorithm, called generation, new solutions are generated by applying crossover and mutation to randomly selected solutions in the population (according to a uniform distribution). Local search is applied again to improve each new solution. The new population is created by merging the best solutions in the population and the new improved solutions. It is worth mentioning that the authors consider four different crossover operators: DPX (similar distance from parents), CX (cycle crossover), OB (order based crossover) and RANK (computing the average ranking of the elements). In computational experiments $\mathrm{CX}$ and $\mathrm{OB}$ performed best.

In [19] a similar method based on combining a classical GA with a local search is presented. It is called hybrid genetic algorithm (HGA) and it is very similar to the method in [31]. The local search is also based on exchanges. Additionally, this method also applies the CX and OB crossover operators. However, instead of DPX and RANK, it applies PMX (partially matched crossover). 
3.8 SA - Simulated Annealing

Simulated annealing proceeds in the same way as ordinary local search, but incorporates some randomization in move selection to avoid getting trapped in a local optimum by means of non-improving moves. The moves are accepted according to probabilities taken from the analogy with the annealing process. As far as we know there is no previous implementation of SA for the LOP although some methods, such as the noising algorithm by Charon and Hudry [7] are based on the same principle. We implemented an SA method based on insertion moves like the other local search based metaheuristics in this section.

\subsection{ILS - Iterated Local Search}

In [31] an iterated local search algorithm is proposed. This method iterates between local search phases by applying three main steps: perturb a locally optimal solution with exchange moves, apply the local search based on insertions to the perturbed solution, and determine the new solution to be perturbed based on the search history. The authors perform a comparison in this paper which favors the memetic algorithm.

\section{The library of benchmark problems}

We have compiled a comprehensive set of benchmark instances including all problem instances that have so far been used for conducting computational experiments. Furthermore we have included new instances. In their original definition, some problem instances are not in normal form. For the computations documented here, all instances have been transformed to normal form. We give a brief description of the origin and the characteristics of the groups of instances.

\subsection{Input/Output matrices}

This is a well-known set of instances, first used in [18]. It contains 50 real-world linear ordering problems taken from input-output tables from various sources. They are comparatively easy and are thus more of interest for economists than for the assessment of approximate methods for hard problems. The original entries in these tables were not necessarily integral, but for the Linear Ordering Library LOLIB they were scaled to integral values.

\subsection{SGB instances}

These instances were used in [24] and are taken from the Stanford GraphBase [23]. They are random instances with entries drawn uniformly distributed from [0,25000]. The set has a total of 25 instances with $n=75$. 
4.3 Random instances of type A

This is a set with 175 random instances that has been widely used for experiments. Problems of type I (called RandomAI) are generated from a $[0,100]$ uniform distribution. This type of problems was proposed in [29] and generated in [5]. Problems were originally generated from a [0, 25000] uniform distribution in [24] and modified afterwards, sampling from a significatively more narrow range (i.e., $[0,100])$ to make them harder to solve. Sizes are $n=100$, 150 , and 200, and there are 25 instances in each set giving a total of 75 . We have extended this set including 25 additional instances with size $n=500$.

Instances of type II (called RandomAII) are generated by counting the number of times a sector appears in a higher position than another in a set of randomly generated permutations. This type of instances was proposed in [6] and generated in [5]. For a problem of size $n, \frac{n}{2}$ permutations are generated. There are 25 instances for each $n$, where $n=100,150$, and 200 .

\subsection{Random instances of type B}

For these random instances, the superdiagonal entries are drawn uniformly distributed from $\left[0, U_{1}\right]$ and the subdiagonal entries from $\left[0, U_{2}\right]$, where $U_{1} \geq U_{2}$. For the problems p40-i with $n=40$, we set $U_{1}=100$ and $U_{2}=100+4(i-1)$. For $n=44$ and 50 we set $U_{1}=100$ and $U_{2}=100+2(i-1)$ for the problems p44-i and p50-i, respectively.

\subsection{Instances of Mitchell and Borchers}

These instances have been used in [28]. They are random matrices where the subdiagonal entries are uniformly distributed in $[0,99]$ and the superdiagonal entries are drawn uniformly from [0,39]. Finally, a certain percentage of the entries was set to zero.

\subsection{Instances of Schiavinotto and Stützle}

Some further benchmark instances have been used in [31]. These instances were generated from the real-world input-output tables of 4.1 by replicating them to obtain larger problems. Thus, the distribution of numbers in these instances somehow reflects real input-output tables, but otherwise they behave more like random problems. That data set has been called XLOLIB; instances with $n=150$ and 250 are available. For each original input-output instance, two instances, one of size $n=150$ and another one of size $n=250$ were generated. The original set contains 98 instances (49 with size 150 and 49 with size 250). We have removed 20 of these instances because there entries were so large that the sum of entries was not representable as a four byte integer. Therefore, this set finally has 78 instances. 
Table 1 Number of instances in each set

\begin{tabular}{|l|rrr|}
\hline Set & \#Instances & \#Optima & \#Upper Bounds \\
\hline IO & 50 & 50 & - \\
SGB & 25 & 25 & - \\
RandomAI & 100 & - & 100 \\
RandomAII & 75 & 25 & 50 \\
RandomB & 90 & 70 & 20 \\
MB & 30 & 30 & - \\
XLOLIB & 78 & - & 78 \\
Special & 36 & 29 & 6 \\
\hline Total & 484 & 229 & 255 \\
\hline
\end{tabular}

4.7 Further special instances

We included some further problem instances that were used for experiments in some publications.

- EX instances

These instances were used in particular in [9] and [10].

- econ instances

The problems instances econ36 through econ77 were generated from the matrix usa79. They turned out not to be solvable as linear program using only 3-dicycle inequalities.

- atp instances

These instances were created from the results of ATP tennis tournaments in 1993/1994. Nodes correspond to a selection of players and the weight of an $\operatorname{arc}(i, j)$ is the number of victories of player $i$ against player $j$.

- Paley graphs

Paley graphs have been used in [17] to prove results about the acyclic subdigraph polytope. They are a special class of tournaments where adjacency comes from an algebraic definition. They are constructed from the members of a suitable finite field by connecting pairs of elements that differ in a quadratic residue.

Table 1 summarizes the number of instances (\#Instances) in each set described above. Moreover, it specifies the number of instances where the optimum is known (\#Optima) or where only an upper bound is known (\#Upper Bounds). In the computational experiments we call OPT-I to the set of 229 instances where the optimum is known, and UB-I to the set of 255 instances where only an upper bound is known.

LOLIB is available at the web sites

- http://comopt.ifi.uni-heidelberg.de/software/LOLIB

- http://heur.uv.es/optsicom/LOLIB 
Also the constants eliminated by the transformation to normal form can be found there. In the appendix of this paper we list all problems with their optimum solution values or currently known best lower and upper bounds.

\section{Computational Comparison}

We divide our experimentation into three parts according to the classification of the instances and methods introduced in previous sections.

In the first experiment we consider the 229 instances of OPT-I and the simple heuristics described in Section 2. In this experiment for each instance and each method we compute the relative deviation Dev (in percent) between the best solution value Value obtained with the method and the optimal value for that instance. For each method, we also report the number of instances \#Opt for which an optimum solution could be found. In addition, we calculate the so-called score statistic [30] associated with each method. For each instance, the nrank of method $M$ is defined as the number of methods that found a better solution than the one found by $M$. In the event of ties, the methods receive the same nrank, equal to the number of methods strictly better than all of them. The value of Score is the sum of the nrank values for all the instances in the experiment, thus, the lower the Score the better the method.

Tables 2 and 3 report about our results for 7 constructive and 7 improving heuristics respectively on the OPT-I set. We do not report running times in these tables because these methods are very fast and their running times are extremely short (below 1 milisecond).

Table 2 shows results for:

- CW: Chenery and Watanabe algorithm

- AM-0: Aujac and Masson algorithm (output coefficients)

- AM-I: Aujac and Masson algorithm (input coefficients)

- Bcq: Becker algorithm (based on quotients)

- Bcr: Becker algorithm (based on rotations)

- BI1: Best Insertion algorithm (variant 1)

- BI2: Best Insertion algorithm (variant 2)

In Table 3 the results obtained with the following improvement methods (started with a random initial solution) are given:

- LSi: Local Search based on insertions

- 2opt: Local Search based on 2-opt

- 3opt: Local Search based on 3-opt

- LSe: Local Search based on exchanges

- KL1: Kernighan-Lin based on exchanges

- KL2: Kernighan-Lin based on insertions

- LE: Local enumeration

Results in Table 2 clearly indicate that OPT-I instances pose a challenge for the simple heuristics with average percentage deviations ranging from $3.49 \%$ 
Table 2 Constructive methods on OPT-I instances

\begin{tabular}{|lrrrrrrr|}
\hline & CW & AM-O & AM-I & Bcq & Bcr & BI1 & BI2 \\
\hline IO & & & & & & & \\
Dev(\%) & 19.07 & 32.94 & 31.45 & 4.07 & 30.19 & 3.24 & 4.18 \\
Score & 231 & 291 & 266 & 101 & 289 & 89 & 104 \\
\#Opt & 0 & 0 & 0 & 0 & 0 & 0 & 0 \\
\hline SGB & & & & & & & \\
Dev(\%) & 12.83 & 26.15 & 26.15 & 3.57 & 31.56 & 3.89 & 3.03 \\
Score & 100 & 125 & 125 & 54 & 175 & 56 & 40 \\
\#Opt & 0 & 0 & 0 & 0 & 0 & 0 & 0 \\
\hline RandomAII & & & & & & & \\
Dev(\%) & 2.60 & 36.50 & 36.55 & 1.57 & 37.75 & 1.09 & 1.26 \\
Score & 100 & 135 & 136 & 68 & 162 & 34 & 48 \\
\#Opt & 0 & 0 & 0 & 0 & 0 & 0 & 0 \\
\hline RandomB & & & & & & & \\
Dev(\%) & 10.13 & 24.69 & 24.69 & 7.04 & 26.41 & 5.24 & 4.87 \\
Score & 276 & 368 & 368 & 194 & 454 & 124 & 106 \\
\#Opt & 0 & 0 & 0 & 0 & 0 & 0 & 0 \\
\hline MB & & & & & & & \\
Dev(\%) & 8.40 & 43.37 & 43.37 & 2.90 & 40.30 & 2.49 & 2.27 \\
Score & 120 & 178 & 178 & 80 & 154 & 52 & 48 \\
\#Opt & 0 & 0 & 0 & 0 & 0 & 0 & 0 \\
\hline Special & & & & & & & \\
Dev(\%) & 12.05 & 34.16 & 33.11 & 4.58 & 27.86 & 5.01 & 5.41 \\
Score & 110 & 161 & 156 & 56 & 153 & 64 & 65 \\
\#Opt & 1 & 2 & 2 & 2 & 1 & 2 \\
\hline \hline OPT-I & & & & & & & \\
Avg. Dev(\%) & 10.85 & 32.97 & 32.55 & 3.95 & 32.35 & 3.49 & 3.50 \\
Sum \#Opt & 1 & 2 & 2 & 2 & 1 & 2 \\
\hline
\end{tabular}

to $32.97 \%$. On the other hand, the improvement methods are able to obtain better solutions with average percentage deviations (shown in Table 3) ranging from $0.57 \%$ to $2.30 \%$. We have not observed significant differences when applying the improvement method from different initial solutions. For example, as shown in Table 3 the LSi method exhibits a Dev value of $0.16 \%$ on the RandomAII instances when it is started from random solutions. When it is run from the CW or the Bcr solutions, it obtains a Dev value of $0.17 \%$ and $0.18 \%$ respectively.

We applied the non-parametric Friedman test for multiple correlated samples to the best solutions obtained by each of the 7 constructive methods. This test computes, for each instance, the rank value of each method according to solution quality (where rank 7 is assigned to the best method and rank 1 to the worst one). Then, it calculates the average rank values of each method across all the instances solved. If the averages differ greatly, the associated $p$-value or significance will be small. The resulting $p$-value of 0.000 obtained in this experiment clearly indicates that there are statistically significant differences 
Table 3 Improvement methods on OPT-I instances

\begin{tabular}{|c|c|c|c|c|c|c|c|}
\hline & LSi & 2opt & 3opt & LSe & KL1 & KL2 & LE \\
\hline \multicolumn{8}{|l|}{ IO } \\
\hline $\operatorname{Dev}(\%)$ & 1.08 & 0.64 & 0.23 & 1.73 & 1.35 & 4.24 & 0.01 \\
\hline Score & 243 & 181 & 125 & 295 & 239 & 232 & 49 \\
\hline \#Opt & 0 & 1 & 4 & 0 & 1 & 0 & 43 \\
\hline \multicolumn{8}{|l|}{ SGB } \\
\hline $\operatorname{Dev}(\%)$ & 0.16 & 0.81 & 0.53 & 1.35 & 0.63 & 0.28 & 1.09 \\
\hline Score & 42 & 122 & 84 & 154 & 100 & 63 & 135 \\
\hline \#Opt & 1 & 0 & 0 & 0 & 0 & 1 & 0 \\
\hline \multicolumn{8}{|l|}{ RandomAII } \\
\hline $\operatorname{Dev}(\%)$ & 0.16 & 0.77 & 0.38 & 0.62 & 0.61 & 0.09 & 0.54 \\
\hline Score & 46 & 161 & 81 & 134 & 134 & 29 & 112 \\
\hline$\# \mathrm{Opt}$ & 0 & 0 & 0 & 0 & 0 & 0 & 0 \\
\hline \multicolumn{8}{|l|}{ RandomB } \\
\hline $\operatorname{Dev}(\%$ & 0.79 & 4.04 & 2.13 & 3.78 & 3.51 & 0.61 & 3.56 \\
\hline Score & 124 & 400 & 232 & 387 & 359 & 95 & 362 \\
\hline \#Opt & 1 & 0 & 0 & 0 & 0 & 1 & 0 \\
\hline \multicolumn{8}{|l|}{ MB } \\
\hline $\operatorname{Dev}(\%)$ & 0.02 & 0.57 & 0.14 & 3.10 & 0.40 & 0.01 & 0.17 \\
\hline Score & 64 & 178 & 113 & 210 & 149 & 41 & 83 \\
\hline \#Opt & 0 & 0 & 0 & 0 & 0 & 4 & 3 \\
\hline \multicolumn{8}{|l|}{ Special } \\
\hline $\operatorname{Dev}(\%)$ & 1.19 & 3.30 & 2.05 & 3.21 & 2.40 & 0.89 & 3.52 \\
\hline Score & 69 & 144 & 82 & 138 & 120 & 49 & 156 \\
\hline \#Opt & 4 & 2 & 2 & 2 & 3 & 3 & 3 \\
\hline \multicolumn{8}{|l|}{ OPT-I } \\
\hline Avg. Dev(\%) & 0.57 & 1.69 & 0.91 & 2.30 & 1.49 & 1.02 & 1.48 \\
\hline Sum \#Opt & 5 & 3 & 6 & 2 & 4 & 8 & 49 \\
\hline
\end{tabular}

among the 7 methods tested. Specifically, the rank values produced by this test are 6.2 (BI2), 6.2 (BI1), 5.6 (Bcq), 3.9 (CW), 2.2 (AM-I), 2.1 (AM-O) and 1.9 (Bcr). Heuristics BI1, BI2 and Bcq consistently provide the best solutions among the constructive heuristics. Considering that BI1 and BI2 obtain very similar rank values, we compared both with two well-known nonparametric tests for pairwise comparisons: the Wilcoxon test and the Sign test. The former one answers the question: Do the two samples (solutions obtained with BI1 and BI2 in our case) represent two different populations? The resulting $p$-value of 0.857 indicates that the values compared could come from the same method. On the other hand, the Sign test computes the number of instances on which an algorithm supersedes another one. The resulting $p$-value of 0.792 indicates that there is no clear winner between BI1 and BI2 when we consider all the instances in the OPT-I set. If we apply these two pairwise tests to compare BI1 and Bcq we obtain a $p$-value of 0.000 (in both tests) indicating that there are significant differences between these constructive methods.

Regarding the improvement methods, LSi and KL2 seem the best ones, although the differences among methods are smaller than in the constructive 
procedures. The significance level of the Friedman test is 0.000 indicating that there are statistically significant differences among the 7 methods tested, and the rank values in this test are: 5.7 (KL2), 5.4 (LSi), 4.8 (3opt), 4.1 (LE), 3.1 (KL1), 2.8 (2opt) and 2.2 (LSe). Considering that KL2 and LSi obtain very similar rank values, we compared both with the Wilcoxon test and the Sign test. The resulting $p$-value of 0.005 obtained in the former one indicates that the values compared come from different methods (using the typical significance level of $\alpha=0.05$ as the threshold between rejecting or not rejecting the null hypothesis). On the other hand, the resulting $p$-value of 0.000 obtained in the Sign test indicates that KL2 consistently beats LSi when we consider all the instances in the OPT-I set.

In our second experiment we consider the metaheuristics described in Section 3 to solve the OPT-I instances. Table 4 reports the values Dev, \#Opt and Score obtained with the following 10 methods executed for 10 seconds on each instance:

- TS: Tabu Search

- MA: Memetic Algorithm

- VNS: Variable Neighbourhood Search

- SA: Simulated Annealing

- SS: Scatter Search

- GRASP: Greedy ramdomized adaptive search procedure

- ILS: Iterated Local Search

- GA: Genetic Algorithm

- KLM: Kernighan-Lin multi-start

- CKM: Chanas and Kobilansky multi-start

Table 4 shows that most of the metaheuristic algorithms considered are able to obtain all the optimal solutions within the time limit of 10 seconds considered (they actually obtain it in around 1 second). The Friedman test indicates that there are statistically significant difference among the methods although some rank values are very similar: 7.15 (ILS), 7.15 (MA), 7.00 (TS), 6.80 (VNS), 6.38 (GRASP), 6.00 (SS), 5.92 (CKM), 4.30 (KLM), 2.80 (SA) and 1.51 (GA). It must be noted that ILS and MA obtain the optimum value in all the instances but one of OPT-I and TS obtains 215 optima out of 229 instances. As expected, the associated $p$-value of the Wilcoxon and Sign tests when comparing ILS and MA is 1 , indicating that we cannot differentiate between both methods in this case. On the other hand, both pairwise tests provide a $p$-value lower than 0.05 when comparing ILS and TS, indicating that there are significant differences between the results of these two methods (although they present the same average percentage deviation to optima: $0.00 \%$ ). We conclude that instances in OPT-I are easy for the best metaheuristics and therefore not adequate to compare them.

We have also computed the upper bound obtained with the linear programming formulation (LP relaxation) described in Section 1 on the OPT-I instances. The percentage gap between the value of this relaxation and the 
Table 4 Metaheuristic algorithms on OPT-I instances running for 10 seconds

\begin{tabular}{|c|c|c|c|c|c|c|c|c|c|c|}
\hline & TS & MA & VNS & $\mathrm{SA}$ & SS & GRASP & ILS & GA & CKM & KLM \\
\hline \multicolumn{11}{|l|}{ IO } \\
\hline $\operatorname{Dev}(\%)$ & 0.00 & 0.00 & 0.00 & 0.03 & 0.01 & 0.00 & 0.00 & 0.38 & 0.23 & 0.00 \\
\hline Score & 50 & 50 & 50 & 304 & 97 & 58 & 50 & 382 & 373 & 50 \\
\hline$\# \mathrm{Opt}$ & 50 & 50 & 50 & 16 & 42 & 49 & 50 & 9 & 10 & 49 \\
\hline \multicolumn{11}{|l|}{ SGB } \\
\hline $\operatorname{Dev}(\%)$ & 0.00 & 0.00 & 0.00 & 0.03 & 0.00 & 0.00 & 0.00 & 0.76 & 0.01 & 0.00 \\
\hline Score & 25 & 25 & 25 & 222 & 52 & 33 & 25 & 245 & 159 & 77 \\
\hline$\# \mathrm{Opt}$ & 25 & 25 & 25 & 0 & 20 & 23 & 25 & 0 & 7 & 16 \\
\hline \multicolumn{11}{|c|}{ RandAII } \\
\hline $\operatorname{Dev}(\%)$ & 0.00 & 0.00 & 0.00 & 0.08 & 0.01 & 0.01 & .00 & 21.44 & 0.01 & 0.02 \\
\hline Score & 25 & 25 & 53 & 222 & 92 & 136 & 25 & 250 & 102 & 136 \\
\hline \#Opt & 25 & 25 & 19 & 0 & 13 & 5 & 25 & 0 & 11 & 6 \\
\hline \multicolumn{11}{|c|}{ RandB 70} \\
\hline $\operatorname{Dev}(\%)$ & 0.00 & 0.00 & 0.02 & 0.25 & 0.01 & 0.00 & 0.00 & 0.75 & 0.13 & 0.00 \\
\hline Score & 70 & 70 & 109 & 546 & 123 & 70 & 70 & 676 & 381 & 82 \\
\hline \#Opt & 70 & 70 & 64 & 10 & 62 & 70 & 70 & 1 & 28 & 68 \\
\hline \multicolumn{11}{|l|}{ MB } \\
\hline $\operatorname{Dev}(\%)$ & 0.00 & 0.00 & 0.00 & 1.33 & 0.00 & 0.00 & 0.00 & 35.53 & 0.00 & 0.00 \\
\hline Score & 30 & 30 & 30 & 270 & 112 & 80 & 30 & 300 & 41 & 169 \\
\hline \#Opt & 30 & 30 & 30 & 0 & 16 & 21 & 30 & 0 & 28 & 10 \\
\hline \multicolumn{11}{|l|}{ Special } \\
\hline $\operatorname{Dev}(\%)$ & 0.02 & 0.00 & 0.11 & 0.39 & 0.09 & 0.05 & 0.00 & 1.38 & 0.14 & 0.01 \\
\hline Score & 77 & 30 & 75 & 189 & 126 & 98 & 30 & 251 & 158 & 107 \\
\hline$\# \mathrm{Opt}$ & 15 & 28 & 20 & 6 & 12 & 14 & 28 & 4 & 9 & 15 \\
\hline \multicolumn{11}{|l|}{ OPT-I } \\
\hline $\operatorname{Dev}(\%)$ & 0.00 & 0.00 & 0.02 & 0.35 & 0.02 & 0.01 & 0.00 & 10.04 & 0.09 & 0.01 \\
\hline \#Opt & 215 & 228 & 208 & 32 & 165 & 182 & 228 & 14 & 93 & 124 \\
\hline
\end{tabular}

optimal solution value provides a measure of its quality. Specifically, the average gap on each subset of instances is: $0.00 \%$ (IO), $0.00 \%$ (SGB), $0.08 \%$ (RandomAII), $1.47 \%$ (15 RandomB instances with $n \leq 150), 0.00 \%$ (MB) and $0.88 \%$ (Special). We can conclude that the value of the upper bound is close to the optimal value on the OPT-I instances.

In our third experiment we target the 255 instances in UB-I where we only have an upper bound for comparison. We do not consider the simple heuristics anymore (since they already had difficulties to solve the easier problems in OPT-I) and limit this comparison to the metaheuristic algorithms considered above. In this experiment, we first determine the best known value for all the instances in UB-I. According to the previous experiment (Table 4) ILS, MA and TS, seem to be the best methods. We therefore run them for one hour on each instance to determine the best known value BestValue for the 255 instances in UB-I.

We give for each instance and each method the relative deviation D.Best (in percent) between the best solution value Value obtained with the method and the best known value BestValue as well as the relative deviation D.UB (in percent) between Value and the upper bound. For each method, we also 
report the number of instances \#Best for which the value of the solution is equal to BestValue. As in the previous experiment we calculate the score statistic. Tables 5 and 6 report the values of these four statistics on the UB-I instances when running the 10 metaheuristic algorithms for 10 and 600 seconds respectively.

According to the differences among methods observed in Table 5, where the deviations w.r.t. the best solution known range from $0.05 \%$ to $16.15 \%$, we can conclude that the instances in set UB-I are more difficult to solve than those in OPT-I (where the deviations range from $0.00 \%$ to $10.04 \%$ ).

Results in Table 5 show that MA is able to obtain the largest number of best solutions (97 of a total of 255 instances) in short runs (10 seconds). No other method is able to obtain more than 55 best solutions, which clearly indicates the superiority of MA. On the other hand, considering average percentage deviations with respect to the best solutions, the differences among the methods appear to be very small. MA and ILS present on average a deviation of $0.05 \%$ and $0.06 \%$ respectively, while TS, SS and VNS present averages deviations of $0.24 \% 0.27 \%$ and $0.29 \%$, respectively. This indicates that although these methods are not able to match most of the best solution values, they obtain solutions with values very close to the best.

Considering the deviations with respect to the upper bound, most of the methods present similar values ranging from $6.66 \%$ to $7.40 \%$ (with the exception of GA $(21.98 \%))$. The associated $p$-value of the Friedman test is 0.000 indicating that there are differences among the 10 metaheuristic procedures observed in this table. The rank values obtained with this non-parametric test are: 9.4 (MA), 8.9 (ILS) 7.3 (TS), 6.3 (VNS), 6.2 (SS), 4.9 (GRASP), 4.1 (SA), 3.6 (KLM), 3.2 (CKM) and $1.0(\mathrm{GA})$. We perform now pairwise comparison between MA and ILS. The resulting $p$-value of 0.000 obtained in both the Wilcoxon and the Sign tests clearly indicates that there are significant differences between the results of both methods. Similarly, we obtain a $p$-value of 0.000 on both tests when comparing ILS and TS, or MA and TS. 
Table 5 Metaheuristic algorithms on UB-I instances running for 10 seconds

\begin{tabular}{|c|c|c|c|c|c|c|c|c|c|c|}
\hline & TS & MA & VNS & $\mathrm{SA}$ & SS & GRASP & ILS & GA & CKM & KLM \\
\hline \multicolumn{11}{|c|}{ RandAI } \\
\hline D.Best & 0.12 & 0.05 & 0.47 & 1.77 & 0.27 & 0.42 & 0.09 & 10.59 & 0.76 & 0.98 \\
\hline D.UB & 17.81 & 17.75 & 18.10 & 18.88 & 17.92 & 18.05 & 17.79 & 26.28 & 18.34 & 18.45 \\
\hline Score & 269 & 115 & 581 & 741 & 423 & 561 & 200 & 1000 & 818 & 792 \\
\hline \#Best & 5 & 33 & 0 & 0 & 1 & 0 & 17 & 0 & 0 & 0 \\
\hline \multicolumn{11}{|c|}{ RandAII } \\
\hline D.Best & 0.01 & 0.00 & 0.01 & 0.07 & 0.02 & 0.04 & 0.00 & 35.97 & 0.06 & 0.02 \\
\hline D.UB & 0.38 & 0.38 & 0.39 & 0.44 & 0.40 & 0.41 & 0.38 & 36.21 & 0.44 & 0.40 \\
\hline Score & 84 & 26 & 93 & 200 & 133 & 176 & 53 & 216 & 216 & 128 \\
\hline \#Best & 3 & 39 & 8 & 0 & 0 & 0 & 14 & 0 & 0 & 0 \\
\hline \multicolumn{11}{|c|}{ RandB } \\
\hline D.Best & 0.00 & 0.00 & 0.00 & 0.31 & 0.02 & 0.00 & 0.00 & 0.91 & 0.01 & 0.14 \\
\hline D.UB & 3.20 & 3.20 & 3.26 & 3.51 & 3.22 & 3.20 & 3.21 & 4.08 & 3.22 & 3.34 \\
\hline Score & 20 & 20 & 76 & 180 & 57 & 20 & 24 & 195 & 60 & 104 \\
\hline \#Best & 20 & 20 & 11 & 0 & 13 & 20 & 19 & 0 & 12 & 7 \\
\hline \multicolumn{11}{|c|}{ XLOLIB } \\
\hline D.Best & 0.64 & 0.14 & 0.44 & 0.56 & 0.71 & 1.17 & 0.15 & 24.01 & 1.76 & 1.95 \\
\hline D.UB & 3.21 & 2.72 & 3.01 & 3.13 & 3.27 & 3.72 & 2.73 & 25.96 & 4.30 & 4.48 \\
\hline Score & 385 & 126 & 269 & 343 & 398 & 538 & 123 & 780 & 656 & 668 \\
\hline \#Best & 0 & 1 & 0 & 0 & 0 & 0 & 1 & 0 & 0 & 0 \\
\hline \multicolumn{11}{|c|}{ Special } \\
\hline D.Best & 0.45 & 0.05 & 0.52 & 2.07 & 0.35 & 0.68 & 0.06 & 9.29 & 0.40 & 0.45 \\
\hline D.UB & 9.61 & 9.26 & 9.67 & 11.04 & 9.52 & 9.81 & 9.27 & 17.35 & 9.57 & 9.61 \\
\hline Score & 25 & 8 & 32 & 64 & 21 & 32 & 8 & 69 & 21 & 23 \\
\hline \#Best & 3 & 4 & 2 & 0 & 3 & 3 & 4 & 0 & 3 & 3 \\
\hline \multicolumn{11}{|l|}{ UB-I } \\
\hline D.Best & 0.24 & 0.05 & 0.29 & 0.95 & 0.27 & 0.46 & 0.06 & 16.15 & 0.60 & 0.71 \\
\hline D.UB & 6.84 & 6.66 & 6.89 & 7.40 & 6.87 & 7.04 & 6.68 & 21.98 & 7.17 & 7.26 \\
\hline \#Best & 31 & 97 & 21 & 0 & 17 & 23 & 55 & 0 & 15 & 10 \\
\hline
\end{tabular}

Results in Table 6 are in line with those of Table 5 (although as expected, the longer the run time the lower the deviations). The average percentage deviations with respect to the best solutions of $0.05 \%$ achieved with MA in 10 seconds runs is reduced to $0.01 \%$ in the 600 seconds runs. Similarly, the average percentage deviations with respect to the best solutions of $0.06 \%$ and $0.24 \%$ achieved with ILS and TS in 10 seconds runs, drops to $0.03 \%$ and $0.16 \%$ respectively in the 600 seconds runs. On the other hand, MA is able to match 205 best known solutions, while none of the other methods obtains more than 108 best known solutions (which is the case of the ILS method followed by TS with 65 ). These results confirm that MA is the best method followed by ILS and TS. As in the previous experiment, we applied the Friedman test, obtaining a $p$-value of 0.000 and the following rank values: 9.25 (MA), 8.30 (ILS), 7.67 (TS), 5.99 (KLM), 5.57 (VNS), 5.24 (GRASP), 5.02 (SS), 3.58 (SA), 3.22 (CKM) and $1.16(\mathrm{GA})$. Pairwise comparison between best methods (MA and ILS, MA 
Table 6 Metaheuristic algorithms on UB-I instances running for 600 seconds

\begin{tabular}{|c|c|c|c|c|c|c|c|c|c|c|}
\hline & TS & MA & VNS & $\mathrm{SA}$ & SS & GRASP & ILS & $\mathrm{GA}$ & CKM & KLM \\
\hline \multicolumn{11}{|c|}{ RandAI } \\
\hline D.Best & 0.10 & 0.00 & 0.41 & 0.40 & 0.18 & 0.28 & 0.09 & 1.14 & 0.44 & 0.27 \\
\hline D.UB & 17.78 & 17.72 & 18.06 & 18.04 & 17.87 & 17.93 & 17.79 & 18.65 & 18.06 & 17.90 \\
\hline Score & 253 & 103 & 640 & 679 & 418 & 492 & 254 & 895 & 703 & 488 \\
\hline \#Best & 20 & 97 & 0 & 0 & 2 & 2 & 23 & 0 & 0 & 15 \\
\hline \multicolumn{11}{|c|}{ RandAII } \\
\hline D.Best & 0.00 & 0.00 & 0.01 & 0.11 & 0.01 & 0.02 & 0.00 & 0.10 & 0.03 & 0.01 \\
\hline D.UB & 0.38 & 0.38 & 0.38 & 0.49 & 0.39 & 0.39 & 0.38 & 0.47 & 0.40 & 0.38 \\
\hline Score & 102 & 50 & 181 & 485 & 272 & 324 & 139 & 379 & 379 & 190 \\
\hline \#Best & 21 & 50 & 16 & 0 & 2 & 0 & 14 & 0 & 0 & 9 \\
\hline \multicolumn{11}{|l|}{ RandB } \\
\hline D.Best & 0.00 & 0.00 & 0.03 & 0.01 & 0.04 & 0.00 & 0.00 & 0.87 & 0.00 & 0.00 \\
\hline D.UB & 3.20 & 3.20 & 3.24 & 3.21 & 3.24 & 3.20 & 3.20 & 4.05 & 3.20 & 3.20 \\
\hline Score & 20 & 20 & 80 & 79 & 86 & 20 & 20 & 200 & 20 & 20 \\
\hline \#Best & 20 & 20 & 12 & 12 & 11 & 20 & 20 & 0 & 20 & 20 \\
\hline \multicolumn{11}{|c|}{ XLOLIB } \\
\hline D.Best & 0.42 & 0.02 & 0.37 & 0.63 & 0.84 & 0.59 & 0.04 & 2.18 & 1.15 & 0.59 \\
\hline D.UB & 2.99 & 2.61 & 2.94 & 3.20 & 3.40 & 3.16 & 2.62 & 4.70 & 3.70 & 3.15 \\
\hline Score & 298 & 121 & 289 & 426 & 529 & 407 & 113 & 702 & 622 & 438 \\
\hline \#Best & 0 & 33 & 0 & 0 & 0 & 0 & 44 & 0 & 0 & 0 \\
\hline \multicolumn{11}{|c|}{ Special } \\
\hline D.Best & 0.26 & 0.02 & 0.23 & 1.20 & 0.24 & 0.55 & 0.00 & 2.25 & 0.18 & 0.15 \\
\hline D.UB & 9.44 & 9.24 & 9.42 & 10.27 & 9.43 & 9.70 & 9.22 & 11.21 & 9.38 & 9.35 \\
\hline Score & 24 & 9 & 23 & 47 & 24 & 35 & 7 & 70 & 22 & 16 \\
\hline \#Best & 4 & 5 & 3 & 2 & 3 & 3 & 7 & 0 & 3 & 4 \\
\hline \multicolumn{11}{|l|}{ UB-I } \\
\hline D.Best & 0.16 & 0.01 & 0.21 & 0.47 & 0.26 & 0.29 & 0.03 & 1.31 & 0.36 & 0.20 \\
\hline D.UB & 6.76 & 6.63 & 6.81 & 7.04 & 6.87 & 6.88 & 6.64 & 7.82 & 6.95 & 6.80 \\
\hline \#Best & 65 & 205 & 31 & 14 & 18 & 25 & 108 & 0 & 23 & 48 \\
\hline
\end{tabular}

and TS, ILS and TS) result in a $p$-value of 0.000 with the Wilcoxon and Sign tests in all the cases.

Considering all the runs performed with the metaheuristic procedures in the three previous experiments, we applied the Friedman test, obtaining a $p$-value of 0.000 and the following rank values overall: 8.65 (MA), 8.14 (ILS), 7.34 (TS), 6.22 (VNS), 5.75 (SS), 5.48 (GRASP), 5.13 (KLM), 3.54 (CKM), 3.53 (SA) and $1.22(\mathrm{GA})$. We can establish three ordered groups of methods according to this ranking. The best methods are MA, ILS and TS. Acceptable performance is shown by VNS, SS, GRASP and KLM, while SA, CKM and GA would be classified as poor.

The predominant performance of MA is particularly intriguing because most of the elements in MA are present in some of the other methods. In particular SS and GA are also based on generating, improving and combining solutions as MA, and they share the same local search procedure based on insertions as the 
improving method. However, as described in [31], the implementation of the local search in MA is performed with a pivoting rule mechanism that explores the neighborhood of a solution in constant time. This reduces the CPU time of the local search by several orders of magnitude, thus permitting MA to explore a significantly larger number of solutions than the other competing methods. It seems then that the implementation details, specially the incremental computation of the move value, make an important difference in local search based methods.

Table 710 replications of 10 seconds on UB-I instances

\begin{tabular}{|c|c|c|c|c|c|c|c|c|c|}
\hline & \multicolumn{3}{|c|}{ TS } & \multicolumn{3}{|c|}{ MA } & \multicolumn{3}{|c|}{ ILS } \\
\hline & MAX & MIN & AVG & MAX & MIN & AVG & MAX & MIN & AVG \\
\hline \multicolumn{10}{|c|}{ RandAI } \\
\hline D.Best & 0.31 & 0.49 & 0.40 & 0.01 & 0.12 & 0.06 & 0.14 & 0.25 & 0.20 \\
\hline D. UB & 37.50 & 37.61 & 37.56 & 37.32 & 37.38 & 37.35 & 37.39 & 37.47 & 37.43 \\
\hline \multicolumn{10}{|c|}{ RandAII } \\
\hline D.Best & 0.01 & 0.02 & 0.01 & 0.00 & 0.00 & 0.00 & 0.00 & 0.01 & 0.00 \\
\hline D. UB & 0.41 & 0.42 & 0.42 & 0.40 & 0.40 & 0.40 & 0.40 & 0.41 & 0.40 \\
\hline \multicolumn{10}{|c|}{ XLOLIB } \\
\hline D.Best & 0.76 & 1.13 & 0.96 & 0.09 & 0.32 & 0.19 & 0.10 & 0.30 & 0.20 \\
\hline D. UB & 3.58 & 3.95 & 3.79 & 2.94 & 3.16 & 3.03 & 2.95 & 3.14 & 3.04 \\
\hline
\end{tabular}

In our final experiment we test the robustness of the three best methods identified so far: MA, ILS and TS. Specifically, we study in this experiment the behavior of the algorithms when they are replicated (executed several times from different random initial solutions). Table 7 shows the deviations (with respect to best solutions and upper bounds) of the maximum value (worst result) MAX, minimum value (best result) MIN and average value AVG of the three methods under comparison. We perform for each method on each problem instance 10 independent runs of 10 seconds each. We limit here the comparison to the most challenging instances: RandAI, RandAII and XLOLIB.

Results in Table 7 indicate that the three methods are quite robust. We observe very small variations between the MAX and MIN values in this table. Moreover, average values, AVG, are very similar (slightly worse) to those reported on Table 5, which correspond to a single run.

\section{Conclusions}

A computational comparison of 24 methods for the LOP has been presented. Overall, experiments with 484 instances were performed to compare the procedures. This extensive experimentation allows us to confirm that classical heuristics, mostly based on simple ordering rules, only obtain good solutions to relatively easy instances (although we can find large instances in this set). Within these heuristics, it turns out that the improvement methods, even when 
applied to random solutions, clearly outperform the constructive procedures. When we target much more difficult instances, we need to apply complex metaheuristics to obtain high quality solutions. Our experimentation reveals that the memetic algorithm implementation MA seems best suited for this problem closely followed by iterated local search ILS, and with the tabu search TS ranked in third place.

\section{Acknowledgement}

The authors thank T. Schiavinotto and T. Stützle for providing their implementation of the memetic and ILS algorithms [31]. The authors also thank J.M. Gonzalez for his implementation of a genetic algorithm.

This research has been partially supported by the Ministerio de Ciencia e Innovación of Spain (TIN2009-07516).

\section{References}

1. Achatz, H., Kleinschmidt P. and Lambsdorff, J.: Der Corruption Perceptions Index und das Linear Ordering Problem, ORNews 26 (2006), 10-12.

2. Aujac, H.: La hiérarchie des industries dans un tableau des echanges industriels, Rev. Economique 2 (1960).

3. BeCKer, O.: Das Helmstädtersche Reihenfolgeproblem - die Effizienz verschiedener Näherungsverfahren, in: Computer uses in the social sciences, Bericht einer Working Conference des Inst. f. höh. Studien u. wiss. Forsch., Wien, 1967.

4. Boenchendorf, K.: Reihenfolgenprobleme / Mean-flow-time sequencing, Mathematical Systems in Economics 74, Verlagsgruppe Athenäum, Hain, Scriptor, 1982.

5. Campos, V., Glover, F. Laguna, M. and Martí, R.: An experimental evaluation of a scatter search for the linear ordering problem, J. of Global Optimization 21 (2001), $397-414$

6. Chanas, S. And Kobylanski, P.: A new heuristic algorithm solving the linear ordering problem, Computational Optimization and Applications 6 (1996), 191-205.

7. Charon, I. AND Hudry, O.: A survey on the linear ordering problem for weighted or unweighted tournaments, 4OR 5 (2007), 5-60.

8. Chenery, H.B. And WAtanaBe, T.: International comparisons of the structure of production, Econometrica 26 (1958), 487-521.

9. Christof, T.: Low-dimensional 0/1-polytopes and branch-and-cut in combinatorial optimization, Shaker, 1997.

10. Christof, T. And Reinelt, G.: Combinatorial optimization and small polytopes, Top 4 (1996), 1-64.

11. Condorcet, M.J.A.N.: Essai sur l'application de l'analyse à la probabilité des décisions rendues à la pluralité des voix, Paris, 1785.

12. Feo, T. And Resende, M.G.C.: Greedy randomized adaptive search procedures, J. of Global Optimization 2 (1995), 1-27.

13. Garcia, C.G., PÉrez-Brito, D., Campos, V. and Martí, R.: Variable neighborhood search for the linear ordering problem, Computers and Operations Research 33 (2006), 3549-3565.

14. GLOver, F.: Future paths for integer programming and links to artificial intelligence,Computers and Operations Research 13 (1986), 533-549.

15. Glover, F., Klastorin, T., And Klingman, D.: Optimal weighted ancestry relationships, Management Science 20 (1974), 1190-1193.

16. Glover, F. And Laguna, M.: Tabu search, Kluwer Academic Publishers, 1997. 
17. Goemans, M.X. And Hall, L.A.: The strongest facets of the acyclic subgraph polytope are unknown, in: Proc. of the 5th Int. IPCO Conference, LNCS 1084, Springer, 1996, 415-429.

18. Grötschel, M., Jünger, M. And Reinelt, G.: A cutting plane algorithm for the linear ordering problem, Operations Research 32 (1984), 1195-1220.

19. Huang, G. And Lim, A.: Designing a hybrid genetic algorithm for the linear ordering problem, in: Cantu-Paz, E. et al. (eds.): Proc. of Genetic and Evolutionary Computation - GECCO 2003, LNCS 2723, Springer, 2003, 1053-1064.

20. Hansen, P. And Mladenovic, N.: Variable neighborhood search, in: Glover, F. and Kochenberger, G. (eds.) Handbook of Metaheuristics 2003, 145-184.

21. JÜNGER, M. AND MUtZeL, P.: 2-layer straightline crossing minimization: performance of exact and heuristic algorithms, J. of Graph Algorithms and Applications 1 (1997), $1-25$.

22. Kernighan, B.W. And Lin, S.: An efficient heuristic procedure for partitioning graphs, Bell Systems Technical Journal 49 (1979) 291-308.

23. Knuth, D.E.: The Stanford GraphBase: a platform for combinatorial computing, Addison-Wesley, 1993.

24. Laguna, M., MaRTí R. AND CAmpos, V.: Intensification and diversification with elite tabu search solutions for the linear ordering problem, Computers and Operations Research 26 (1999), 1217-1230.

25. Laguna, M. And Martí R.: Scatter search: methodology and implementations in $C$, Kluwer Academic Publishers, 2003.

26. LEONTIEF, W.: Quantitative input-output relations in the economic system of the United States, The Review of Economics and Statistics 18 (1936).

27. MaRtí R. And Reinelt, G.: The linear ordering problem: exact and heuristics methods in combinatorial optimization, Applied Mathematical Sciences 175, Springer, 2010.

28. Mitchell, J.E. And Borchers, B.: Solving linear ordering problems, in: Frenk, H., Roos, K., Terlaky, T. and Zhang, S. (eds.):, High Performance Optimization, Applied Optimization Vol. 33, Kluwer, 2000, 340-366.

29. Reinelt, G.: The linear ordering problem: algorithms and applications, Research and Exposition in Mathematics 8, Heldermann, 1985.

30. Ribeiro, C.C.. UCHOA, E. AND Werneck, R.F.: A Hybrid GRASP with Perturbations for the Steiner Problem in Graphs, INFORMS Journal on Computing 14 (2002), 228 246.

31. Schindinotto, T. And Stützle, T.: The linear ordering problem: Instances, search space analysis and algorithms, J. of Mathematical Modelling and Algorithms 3 (2004), 367-402. 


\section{Appendix}

We list all problem instances with optimum solution values (printed in bold) or best known lower and upper bounds (in brackets). Note that after having conducted the experiments for this paper a small number of additional optima have been proved. But this does not affect the conclusions drawn from our computations.

\begin{tabular}{|r|r|r|r|}
\hline Problem & Bounds & Problem & Bounds \\
\hline be75eec & $\mathbf{2 3 6 4 6 4}$ & be75np & $\mathbf{7 1 6 9 9 4}$ \\
be75oi & $\mathbf{1 1 1 1 7 1}$ & be75tot & $\mathbf{9 8 0 5 1 6}$ \\
stabu70 & $\mathbf{3 6 2 5 1 2}$ & stabu74 & $\mathbf{5 4 1 3 9 3}$ \\
stabu75 & $\mathbf{5 5 3 3 0 3}$ & t59b11xx & $\mathbf{2 0 9 3 2 0}$ \\
t59d11xx & $\mathbf{1 4 7 3 5 4}$ & t59f11xx & $\mathbf{1 2 2 5 2 0}$ \\
t59i11xx & $\mathbf{8 2 6 1 5 4 5}$ & t59n11xx & $\mathbf{2 0 9 2 8}$ \\
t65b11xx & $\mathbf{3 5 6 7 5 8}$ & t65d11xx & $\mathbf{2 3 7 7 3 9}$ \\
t65f11xx & $\mathbf{2 1 7 2 9 5}$ & t65i11xx & $\mathbf{1 4 4 6 9 1 6 3}$ \\
t65111xx & $\mathbf{1 6 7 1 9}$ & t65n11xx & $\mathbf{3 2 1 5 7}$ \\
t65w11xx & $\mathbf{1 3 8 1 8 1 0 2 9}$ & t69r11xx & $\mathbf{7 7 1 1 4 9}$ \\
t70b11xx & $\mathbf{5 2 8 4 1 9}$ & t70d11xx & $\mathbf{3 7 6 7 2 5}$ \\
t70d11xxb & $\mathbf{3 6 6 4 6 9}$ & t70f11xx & $\mathbf{3 6 0 3 3 6}$ \\
t70i11xx & $\mathbf{2 4 7 8 5 7 8 2}$ & t70k11xx & $\mathbf{6 0 6 5 9 2 0 0}$ \\
t70111xx & $\mathbf{2 5 2 5 3}$ & t70n11xx & $\mathbf{5 2 7 0 4}$ \\
t70u11xx & $\mathbf{2 1 7 1 6 4 0 0}$ & t70w11xx & $\mathbf{2 2 4 3 1 9 9 5 4}$ \\
t70x11xx & $\mathbf{2 8 3 8 0 8 8 6 5}$ & t74d11xx & $\mathbf{5 6 6 0 8 9}$ \\
t75d11xx & $\mathbf{5 7 8 3 0 4}$ & t75e11xx & $\mathbf{2 7 3 9 2 1 9}$ \\
t75i11xx & $\mathbf{6 3 5 6 7 7 3 5}$ & t75k11xx & $\mathbf{1 0 8 8 4 4}$ \\
t75n11xx & $\mathbf{9 3 7 7 7}$ & t75u11xx & $\mathbf{5 2 7 0 8 3 2 3}$ \\
tiw56n54 & $\mathbf{9 1 5 5 4}$ & tiw56n58 & $\mathbf{1 2 5 2 2 4}$ \\
tiw56n62 & $\mathbf{1 7 6 7 1 5}$ & tiw56n66 & $\mathbf{2 2 6 5 4 7}$ \\
tiw56n67 & $\mathbf{2 2 6 0 3 3}$ & tiw56n72 & $\mathbf{3 6 5 1 4 6}$ \\
tiw56r54 & $\mathbf{1 0 2 9 4 8}$ & tiw56r58 & $\mathbf{1 2 9 5 6 8}$ \\
tiw56r66 & $\mathbf{2 0 9 4 9 1 ~}$ & tiw56r67 & $\mathbf{2 2 2 8 1 0}$ \\
tiw56r72 & $\mathbf{2 7 0 6 6 3}$ & usa79 & $\mathbf{1 8 1 3 9 8 6}$ \\
\hline
\end{tabular}

Table 8 Bounds for IO problems

\begin{tabular}{|r|r|r|r|}
\hline Problem & Bounds & Problem & Bounds \\
\hline sgb75.01 & $\mathbf{2 7 2 4 1 2 6}$ & sgb75.02 & $\mathbf{2 6 1 6 3 9 2}$ \\
sgb75.03 & $\mathbf{2 7 4 7 3 8 4}$ & sgb75.04 & $\mathbf{2 7 3 4 1 6 9}$ \\
sgb75.05 & $\mathbf{2 7 0 7 8 6 3}$ & sgb75.06 & $\mathbf{2 7 0 7 2 8 0}$ \\
sgb75.07 & $\mathbf{2 7 2 7 9 2 8}$ & sgb75.08 & $\mathbf{2 7 1 2 8 3 7}$ \\
sgb75.09 & $\mathbf{2 6 8 7 3 6 4}$ & sgb75.10 & $\mathbf{2 7 3 3 3 8 7}$ \\
sgb75.11 & $\mathbf{2 7 3 2 6 8 6}$ & sgb75.12 & $\mathbf{2 6 9 2 5 4 8}$ \\
sgb75.13 & $\mathbf{2 7 1 4 5 9 1}$ & sgb75.14 & $\mathbf{2 7 3 3 9 2 6}$ \\
sgb75.15 & $\mathbf{2 7 3 2 8 1 0}$ & sgb75.16 & $\mathbf{2 7 4 7 7 9 7}$ \\
sgb75.17 & $\mathbf{2 7 4 7 8 6 4}$ & sgb75.18 & $\mathbf{2 5 7 9 3 4 4}$ \\
sgb75.19 & $\mathbf{2 7 3 6 2 2 1}$ & sgb75.20 & $\mathbf{2 7 3 2 0 2 1}$ \\
sgb75.21 & $\mathbf{2 7 4 0 2 8 9}$ & sgb75.22 & $\mathbf{2 7 1 0 1 2 2}$ \\
sgb75.23 & $\mathbf{2 7 2 0 9 8 1}$ & sgb75.24 & $\mathbf{2 7 4 3 8 7 9}$ \\
sgb75.25 & $\mathbf{2 7 3 1 5 4 2}$ & & \\
\hline
\end{tabular}

Table 9 Bounds for SGB problems 


\begin{tabular}{|c|c|c|c|}
\hline Problem & Bounds & Problem & Bounds \\
\hline t1d100.01 & {$[106852,114468]$} & t1d100.02 & {$[105947,114077]$} \\
\hline $\mathrm{t} 1 \mathrm{~d} 100.03$ & {$[109819,117843]$} & $\mathrm{t} 1 \mathrm{~d} 100.04$ & {$[109252,117639]$} \\
\hline t1d 100.05 & {$[108859,117538]$} & $\mathrm{t} 1 \mathrm{~d} 100.06$ & {$[108201,117057]$} \\
\hline t1d 100.07 & {$[108803,117118]$} & $\mathrm{t} 1 \mathrm{~d} 100.08$ & {$[107480,115756]$} \\
\hline t1d 100.09 & {$[108549,116527]$} & $\mathrm{t} 1 \mathrm{~d} 100.10$ & {$[108771,117518]$} \\
\hline $\mathrm{t} 1 \mathrm{~d} 100.11$ & {$[107920,116637]$} & $\mathrm{t} 1 \mathrm{~d} 100.12$ & {$[108389,116617]$} \\
\hline $\mathrm{t} 1 \mathrm{~d} 100.13$ & {$[108702,116816]$} & $\mathrm{t} 1 \mathrm{~d} 100.14$ & {$[105583,114199]$} \\
\hline $\mathrm{t} 1 \mathrm{~d} 100.15$ & {$[108667,117221]$} & $\mathrm{t} 1 \mathrm{~d} 100.16$ & {$[107426,115781]$} \\
\hline $\mathrm{t} 1 \mathrm{~d} 100.17$ & {$[105612,113860]$} & $\mathrm{t} 1 \mathrm{~d} 100.18$ & {$[107861,115959]$} \\
\hline t1d 100.19 & {$[108026,116987]$} & $\mathrm{t} 1 \mathrm{~d} 100.20$ & {$[109968,119008]$} \\
\hline $\mathrm{t} 1 \mathrm{~d} 100.21$ & {$[107255,116326]$} & $\mathrm{t} 1 \mathrm{~d} 100.22$ & {$[108250,116987]$} \\
\hline $\mathrm{t} 1 \mathrm{~d} 100.23$ & {$[106146,113264]$} & t1d 100.24 & {$[108782,116959]$} \\
\hline $\mathrm{t} 1 \mathrm{~d} 100.25$ & {$[106933,115311]$} & t1d 150.01 & {$[235056,258334]$} \\
\hline t1d150.02 & {$[234421,261964]$} & t1d150.03 & {$[236319,261648]$} \\
\hline t1d150.04 & {$[234506,260037]$} & t1d150.05 & {$[234601,260155]$} \\
\hline t1d150.06 & {$[234465,257685]$} & t1d 150.07 & {$[235283,257133]$} \\
\hline t1d 150.08 & {$[237230,264958]$} & t1d150.09 & {$[237253,258980]$} \\
\hline t1d 150.10 & {$[234821,260359]$} & t1d150.11 & {$[234157,259316]$} \\
\hline $\mathrm{t} 1 \mathrm{~d} 150.12$ & {$[236318,262105]$} & t1d150.13 & {$[237116,262727]$} \\
\hline $\mathrm{t} 1 \mathrm{~d} 150.14$ & {$[234453,259552]$} & t1d 150.15 & {$[232065,257699]$} \\
\hline $\mathrm{t} 1 \mathrm{~d} 150.16$ & {$[232948,258626]$} & $\mathrm{t} 1 \mathrm{~d} 150.17$ & {$[236656,262929]$} \\
\hline $\mathrm{t} 1 \mathrm{~d} 150.18$ & {$[234348,259865]$} & t1d150.19 & {$[234994,260286]$} \\
\hline t1d 150.20 & {$[235411,260652]$} & $\mathrm{t} 1 \mathrm{~d} 150.21$ & {$[233980,259087]$} \\
\hline t1d 150.22 & {$[235415,260134]$} & $\mathrm{t} 1 \mathrm{~d} 150.23$ & {$[233492,258077]$} \\
\hline t1d150.24 & {$[236016,261151]$} & t1d150.25 & [236428, 261299] \\
\hline $\mathrm{t} 1 \mathrm{~d} 200.01$ & {$[410871,459555]$} & t1d200.02 & {$[407729,461223]$} \\
\hline $\mathrm{t} 1 \mathrm{~d} 200.03$ & {$[407420,459905]$} & t1d200.04 & {$[410101,465271]$} \\
\hline $\mathrm{t} 1 \mathrm{~d} 200.05$ & {$[411522,462358]$} & $\mathrm{t} 1 \mathrm{~d} 200.06$ & {$[406451,461499]$} \\
\hline $\mathrm{t} 1 \mathrm{~d} 200.07$ & {$[412482,460054]$} & $\mathrm{t} 1 \mathrm{~d} 200.08$ & {$[408850,462476]$} \\
\hline t1d200.09 & {$[409308,457683]$} & $\mathrm{t} 1 \mathrm{~d} 200.10$ & {$[406453,467356]$} \\
\hline $\mathrm{t} 1 \mathrm{~d} 200.11$ & {$[410239,462167]$} & $\mathrm{t} 1 \mathrm{~d} 200.12$ & {$[412831,465075]$} \\
\hline $\mathrm{t} 1 \mathrm{~d} 200.13$ & [409234, 459659] & $\mathrm{t} 1 \mathrm{~d} 200.14$ & {$[408879,458933]$} \\
\hline $\mathrm{t} 1 \mathrm{~d} 200.15$ & {$[409061,463328]$} & $\mathrm{t} 1 \mathrm{~d} 200.16$ & {$[408059,463822]$} \\
\hline $\mathrm{t} 1 \mathrm{~d} 200.17$ & {$[410280,461326]$} & $\mathrm{t} 1 \mathrm{~d} 200.18$ & {$[407709,467958]$} \\
\hline $\mathrm{t} 1 \mathrm{~d} 200.19$ & {$[412947,455589]$} & $\mathrm{t} 1 \mathrm{~d} 200.20$ & {$[406418,455487]$} \\
\hline $\mathrm{t} 1 \mathrm{~d} 200.21$ & {$[408037,469845]$} & $\mathrm{t} 1 \mathrm{~d} 200.22$ & {$[407333,458596]$} \\
\hline $\mathrm{t} 1 \mathrm{~d} 200.23$ & {$[408552,460653]$} & $\mathrm{t} 1 \mathrm{~d} 200.24$ & {$[410583,459286]$} \\
\hline $\mathrm{t} 1 \mathrm{~d} 200.25$ & {$[406356,458197]$} & t1d500.01 & {$[2402774,4191813]$} \\
\hline t1d500.02 & {$[2411570,4207198]$} & t $1 d 500.03$ & {$[2404784,4205918]$} \\
\hline t1d500.04 & {$[2413600,4221950]$} & t1d500.05 & {$[2391486,4186810]$} \\
\hline t1d500.06 & {$[2399394,4190956]$} & t1d500.07 & {$[2400739,4198457]$} \\
\hline $\mathrm{t} 1 \mathrm{~d} 500.08$ & {$[2413108,4206654]$} & t1d500.09 & {$[2406343,4198840]$} \\
\hline $\mathrm{t} 1 \mathrm{~d} 500.10$ & {$[2404420,4198760]$} & $\mathrm{t} 1 \mathrm{~d} 500.11$ & {$[2416364,4210737]$} \\
\hline $\mathrm{t} 1 \mathrm{~d} 500.12$ & {$[2402581,4194185]$} & $\mathrm{t} 1 \mathrm{~d} 500.13$ & {$[2405118,4197442]$} \\
\hline $\mathrm{t} 1 \mathrm{~d} 500.14$ & {$[2410693,4200887]$} & t $1 d 500.15$ & {$[2411718,4208905]$} \\
\hline $\mathrm{t} 1 \mathrm{~d} 500.16$ & {$[2416067,4200206]$} & $\mathrm{t} 1 \mathrm{~d} 500.17$ & {$[2401800,4197344]$} \\
\hline $\mathrm{t} 1 \mathrm{~d} 500.18$ & {$[2421159,4222286]$} & t1d500.19 & {$[2404029,4198658]$} \\
\hline $\mathrm{t} 1 \mathrm{~d} 500.20$ & {$[2414713,4207789]$} & $\mathrm{t} 1 \mathrm{~d} 500.21$ & {$[2405615,4201350]$} \\
\hline $\mathrm{t} 1 \mathrm{~d} 500.22$ & {$[2408164,4208557]$} & $\mathrm{t} 1 \mathrm{~d} 500.23$ & {$[2408689,4197731]$} \\
\hline t1d500.24 & {$[2402712,4191909]$} & t1d500.25 & {$[2405718,4196590]$} \\
\hline
\end{tabular}

Table 10 Bounds for RandA1 problems 


\begin{tabular}{|c|c|c|c|}
\hline Problem & Bounds & Problem & Bounds \\
\hline t2d100.01 & 25362 & t2d100.02 & 28326 \\
\hline $\mathrm{t} 2 \mathrm{~d} 100.03$ & 25886 & $\mathrm{t} 2 \mathrm{~d} 100.04$ & 26076 \\
\hline t2d100.05 & 25118 & $\mathrm{t} 2 \mathrm{~d} 100.06$ & 25380 \\
\hline t2d100.07 & 27144 & t2d100.08 & 23784 \\
\hline t2d100.09 & 27752 & $\mathrm{t} 2 \mathrm{~d} 100.10$ & 26690 \\
\hline $\mathrm{t} 2 \mathrm{~d} 100.11$ & 25106 & $\mathrm{t} 2 \mathrm{~d} 100.12$ & 26782 \\
\hline $\mathrm{t} 2 \mathrm{~d} 100.13$ & 27878 & $\mathrm{t} 2 \mathrm{~d} 100.14$ & 25878 \\
\hline t $2 \mathrm{~d} 100.15$ & 24232 & $\mathrm{t} 2 \mathrm{~d} 100.16$ & 28206 \\
\hline $\mathrm{t} 2 \mathrm{~d} 100.17$ & 26704 & $\mathrm{t} 2 \mathrm{~d} 100.18$ & 26928 \\
\hline $\mathrm{t} 2 \mathrm{~d} 100.19$ & 28760 & t $2 \mathrm{~d} 100.20$ & 25220 \\
\hline t2d100.21 & 24452 & t $2 \mathrm{~d} 100.22$ & 27230 \\
\hline t2d100.23 & 25588 & t2d100.24 & 24800 \\
\hline t $2 \mathrm{~d} 100.25$ & 23742 & t2d150.01 & {$[76041,76276]$} \\
\hline t2d150.02 & {$[73624,73811]$} & t2d150.03 & {$[69705,69894]$} \\
\hline t2d150.04 & {$[73963,74136]$} & t2d150.05 & {$[79723,79847]$} \\
\hline t2d150.06 & {$[75440,75604]$} & t2d150.07 & {$[73858,74067]$} \\
\hline t2d150.08 & {$[67463,67803]$} & t2d150.09 & {$[70739,70915]$} \\
\hline t $2 \mathrm{~d} 150.10$ & {$[69029,69302]$} & $\mathrm{t} 2 \mathrm{~d} 150.11$ & {$[72800,72987]$} \\
\hline t2d150.12 & {$[72181,72429]$} & $\mathrm{t} 2 \mathrm{~d} 150.13$ & {$[74580,74739]$} \\
\hline t2d150.14 & [68132, 68331] & t $2 \mathrm{~d} 150.15$ & {$[76831,77007]$} \\
\hline t2d150.16 & {$[72018,72238]$} & $\mathrm{t} 2 \mathrm{~d} 150.17$ & {$[70185,70468]$} \\
\hline t2d150.18 & {$[73191,73424]$} & $\mathrm{t} 2 \mathrm{~d} 150.19$ & {$[75958,76133]$} \\
\hline t $2 \mathrm{~d} 150.20$ & {$[67370,67651]$} & $\mathrm{t} 2 \mathrm{~d} 150.21$ & {$[70297,70571]$} \\
\hline t2d150.22 & {$[69287,69515]$} & $\mathrm{t} 2 \mathrm{~d} 150.23$ & {$[74799,74958]$} \\
\hline t2d150.24 & {$[70063,70323]$} & $\mathrm{t} 2 \mathrm{~d} 150.25$ & {$[73853,74026]$} \\
\hline t2d200.01 & {$[147742,148294]$} & t2d 200.02 & {$[144218,144903]$} \\
\hline t2d200.03 & {$[141378,142105]$} & t2d200.04 & {$[150878,151471]$} \\
\hline t2d200.05 & {$[150236,150854]$} & $\mathrm{t} 2 \mathrm{~d} 200.06$ & {$[141254,142009]$} \\
\hline t2d 200.07 & {$[149752,150379]$} & $\mathrm{t} 2 \mathrm{~d} 200.08$ & {$[149910,150415]$} \\
\hline t2d200.09 & {$[141958,142747]$} & $\mathrm{t} 2 \mathrm{~d} 200.10$ & {$[149630,150232]$} \\
\hline t2d200.11 & {$[147542,148262]$} & $\mathrm{t} 2 \mathrm{~d} 200.12$ & {$[152470,153064]$} \\
\hline $\mathrm{t} 2 \mathrm{~d} 200.13$ & {$[137618,138514]$} & $\mathrm{t} 2 \mathrm{~d} 200.14$ & {$[144384,145083]$} \\
\hline t2d200.15 & {$[140442,141227]$} & $\mathrm{t} 2 \mathrm{~d} 200.16$ & {$[147448,148114]$} \\
\hline t2d 200.17 & {$[131874,132728]$} & t2d200.18 & {$[151196,151663]$} \\
\hline t2d 200.19 & {$[137314,138182]$} & t $2 \mathrm{~d} 200.20$ & {$[146508,147051]$} \\
\hline t2d200.21 & {$[143568,144221]$} & $\mathrm{t} 2 \mathrm{~d} 200.22$ & {$[146920,147524]$} \\
\hline t $2 \mathrm{~d} 200.23$ & {$[145034,145731]$} & $\mathrm{t} 2 \mathrm{~d} 200.24$ & {$[151260,151769]$} \\
\hline t2d200.25 & {$[149128,149723]$} & & \\
\hline
\end{tabular}

Table 11 Bounds for RandA2 problems 


\begin{tabular}{|c|c|c|c|}
\hline Problem & Bounds & Problem & Bounds \\
\hline$p 40-01$ & 29457 & $\mathrm{p} 40-02$ & 27482 \\
\hline p40-03 & 28061 & p40-04 & 28740 \\
\hline p40-05 & 27450 & $\mathrm{p} 40-06$ & 29164 \\
\hline p 40-07 & 28379 & $\mathrm{p} 40-08$ & 28267 \\
\hline p40-09 & 30578 & p40-10 & 31737 \\
\hline p40-11 & 30658 & $\mathrm{p} 40-12$ & 30986 \\
\hline p40-13 & 33903 & p40-14 & 34078 \\
\hline p 40-15 & 34659 & p40-16 & 36044 \\
\hline $\mathrm{p} 40-17$ & 38201 & p40-18 & 37562 \\
\hline p40-19 & 38956 & $\mathrm{p} 40-20$ & 39658 \\
\hline p44-01 & 35948 & p44-02 & 35314 \\
\hline p44-03 & 34335 & p44-04 & 33551 \\
\hline p44-05 & 34827 & $\mathrm{p} 44-06$ & 33962 \\
\hline p44-07 & 33171 & p44-08 & 34127 \\
\hline p44-09 & 33403 & p44-10 & 33778 \\
\hline p44-11 & 34016 & p44-12 & 33850 \\
\hline p $44-13$ & 35385 & p $44-14$ & 35801 \\
\hline p44-15 & 33827 & p44-16 & 36188 \\
\hline p44-17 & 35454 & p44-18 & 36669 \\
\hline p44-19 & 36436 & $\mathrm{p} 44-20$ & 37438 \\
\hline p44-21 & 37786 & p44-22 & 36722 \\
\hline $\mathrm{p} 44-23$ & 36605 & p44-24 & 38286 \\
\hline $\mathrm{p} 44-25$ & 38129 & $\mathrm{p} 44-26$ & 39107 \\
\hline p 44-27 & 39170 & $\mathrm{p} 44-28$ & 40264 \\
\hline p 44-29 & 41819 & $\mathrm{p} 44-30$ & 40387 \\
\hline $\mathrm{p} 44-31$ & 43817 & p44-32 & 42545 \\
\hline p 44-33 & 42355 & $\mathrm{p} 44-34$ & 44988 \\
\hline $\mathrm{p} 44-35$ & 44114 & $\mathrm{p} 44-36$ & 45575 \\
\hline $\mathrm{p} 44-37$ & 45297 & $\mathrm{p} 44-38$ & 47414 \\
\hline $\mathrm{p} 44-39$ & 48979 & $\mathrm{p} 44-40$ & 47774 \\
\hline p44-41 & 48137 & p44-42 & 49511 \\
\hline p 44-43 & 51014 & p44-44 & 51949 \\
\hline p 44-45 & 52857 & $\mathrm{p} 44-46$ & 52776 \\
\hline p44-47 & 54122 & $\mathrm{p} 44-48$ & 54355 \\
\hline $\mathrm{p} 44-49$ & 57279 & $\mathrm{p} 44-50$ & 56444 \\
\hline p50-01 & 44667 & p50-02 & {$[43835,44866]$} \\
\hline p50-03 & {$[44256,45257]$} & p50-04 & {$[43928,45026]$} \\
\hline p50-05 & {$[42907,44196]$} & p50-06 & {$[42325,43765]$} \\
\hline p50-07 & {$[42640,43977]$} & p50-08 & {$[42666,44655]$} \\
\hline p50-09 & {$[43711,45183]$} & p50-10 & {$[43575,45346]$} \\
\hline p50-11 & {$[43527,45132]$} & p50-12 & {$[42809,44671]$} \\
\hline p50-13 & {$[43169,44872]$} & p50-14 & {$[44519,46272]$} \\
\hline p50-15 & {$[44866,46479]$} & p50-16 & {$[45310,46693]$} \\
\hline p50-17 & {$[46011,47751]$} & p50-18 & {$[46897,48152]$} \\
\hline p50-19 & {$[47212,49162]$} & p50-20 & {$[46779,48155]$} \\
\hline
\end{tabular}

Table 12 Bounds for RandB problems 


\begin{tabular}{|r|r|r|r|}
\hline Problem & Bounds & Problem & Bounds \\
\hline r100a 2 & $\mathbf{1 4 5 2 7 0}$ & r100b2 & $\mathbf{1 4 3 2 7 1}$ \\
r100c2 & $\mathbf{1 4 1 7 0 2}$ & r100d2 & $\mathbf{1 4 2 6 3 0}$ \\
r100e2 & $\mathbf{1 4 7 4 1 6}$ & r150a0 & $\mathbf{3 6 0 9 7 8}$ \\
r150a1 & $\mathbf{3 4 9 2 5 1}$ & r150b0 & $\mathbf{3 6 7 6 3 5}$ \\
r150b1 & $\mathbf{3 4 7 6 2 7}$ & r150c0 & $\mathbf{3 6 3 8 9 5}$ \\
r150c1 & $\mathbf{3 4 6 4 9 2}$ & r150d0 & $\mathbf{3 6 3 1 8 0}$ \\
r150d1 & $\mathbf{3 4 8 9 0 2}$ & r150e0 & $\mathbf{3 6 7 1 8 1}$ \\
r150e1 & $\mathbf{3 4 9 9 1 0}$ & r200a0 & $\mathbf{6 5 4 6 0 4}$ \\
r200a1 & $\mathbf{6 1 6 3 9 9}$ & r200b0 & $\mathbf{6 5 1 2 3 7}$ \\
r200b1 & $\mathbf{6 2 2 1 1 2}$ & r200c0 & $\mathbf{6 5 7 4 4 1}$ \\
r200c1 & $\mathbf{6 1 1 9 5 6}$ & r200d0 & $\mathbf{6 5 4 3 7 5}$ \\
r200d1 & $\mathbf{6 1 6 6 1 7}$ & r200e0 & $\mathbf{6 4 5 2 0 7}$ \\
r200e1 & $\mathbf{6 1 1 3 0 6}$ & r250a0 & $\mathbf{1 0 1 9 1 2 0}$ \\
r250b0 & $\mathbf{1 0 1 3 7 3 7}$ & r250c0 & $\mathbf{1 0 1 0 9 6 1}$ \\
r250d0 & $\mathbf{1 0 1 5 0 4 1}$ & r250e0 & $\mathbf{1 0 0 8 2 6 7}$ \\
\hline
\end{tabular}

Table 13 Bounds for MB problems

\begin{tabular}{|r|r|r|r|}
\hline Problem & Bounds & Problem & Bounds \\
\hline atp24 & $\mathbf{1 7 2}$ & atp48 & $\mathbf{4 8 3}$ \\
atp66 & $\mathbf{7 6 1}$ & atp76 & $\mathbf{9 3 4}$ \\
atp111 & {$[1495,1636]$} & atp134 & {$[1797,2011]$} \\
atp163 & {$[2073,2417]$} & atp452 & {$[2713,3132]$} \\
econ36 & $\mathbf{5 4 8 5 7 4}$ & econ43 & $\mathbf{6 6 7 3 6 9}$ \\
econ47 & $\mathbf{8 2 8 8 1 6}$ & econ58 & $\mathbf{1 2 2 1 8 8 8}$ \\
econ59 & $\mathbf{1 2 0 9 6 8 3}$ & econ61 & $\mathbf{1 2 1 8 0 2 3}$ \\
econ62 & $\mathbf{1 2 3 5 6 7 7}$ & econ64 & $\mathbf{1 2 7 2 4 6 1}$ \\
econ67 & $\mathbf{1 3 8 8 3 1 7}$ & econ68 & $\mathbf{1 4 3 8 3 7 8}$ \\
econ71 & $\mathbf{1 5 5 8 2 9 2}$ & econ72 & $\mathbf{1 8 3 5 6 3 1}$ \\
econ73 & $\mathbf{2 0 4 6 1 1 2}$ & econ76 & $\mathbf{2 6 4 9 1 3 4}$ \\
econ77 & $\mathbf{2 6 7 4 7 3 2}$ & ex1 & $\mathbf{4 4 9}$ \\
ex2 & $\mathbf{4 4 1}$ & ex3 & $\mathbf{4 3 8}$ \\
ex4 & $\mathbf{3 9 0}$ & ex5 & $\mathbf{4 0 5}$ \\
ex6 & $\mathbf{3 9 5}$ & pal11 & $\mathbf{3 5}$ \\
pal19 & $\mathbf{1 0 7}$ & pal23 & $\mathbf{1 6 1}$ \\
pal27 & $\mathbf{2 5 2}$ & pal31 & {$[285,300]$} \\
pal43 & {$[543,597]$} & pal55 & {$[1045,1084]$} \\
\hline
\end{tabular}

Table 14 Bounds for Spec problems 


\begin{tabular}{|c|c|c|c|}
\hline Problem & Bounds & Problem & Bounds \\
\hline be75eec_150 & {$[3482828,3527035]$} & be75np_150 & {$[7174935,7317546]$} \\
\hline be75oi_150 & {$[2246534,2259482]$} & be75tot_150 & {$[12287707,12509023]$} \\
\hline stabu1_150 & {$[2875732,2923697]$} & stabu2_150 & {$[4327538,4398662]$} \\
\hline stabu3_150 & {$[4510445,4582377]$} & t59b11xx_150 & [3239550, 3298634] \\
\hline t59d11xx_150 & {$[1462697,1500324]$} & t59f $11 \mathrm{xx} \_150$ & {$[1543733,1578719]$} \\
\hline t59n11xx_150 & {$[319001,323636]$} & t65b11xx_150 & {$[6454749,6547627]$} \\
\hline t65d11xx_150 & {$[3559347,3646934]$} & t65f $11 \mathrm{xx} \_150$ & [3159526, 3231148] \\
\hline t65111xx_150 & {$[253396,255390]$} & t65n11xx_150 & {$[550849,558953]$} \\
\hline t69r11xx_150 & {$[11855957,12021166]$} & t70b11xx_150 & {$[9649306,9802850]$} \\
\hline t70d11xn_150 & {$[5825509,5956793]$} & t70d11xx_150 & {$[6174178,6305710]$} \\
\hline t70f $11 \mathrm{xx} \_150$ & {$[5150097,5285191]$} & t70111xx_150 & {$[436862,438087]$} \\
\hline t70n11xx_150 & {$[948913,959803]$} & t74d11xx_150 & {$[9396044,9601749]$} \\
\hline t75d11xx_150 & {$[9644779,9850135]$} & $\mathrm{t} 75 \mathrm{e} 11 \mathrm{xx} \_150$ & {$[41570193,42053463]$} \\
\hline t75k11xx_150 & {$[1541596,1569847]$} & $\mathrm{t} 75 \mathrm{n} 11 \mathrm{xx} \_150$ & {$[1743094,1767716]$} \\
\hline tiw56n54_150 & {$[837945,857599]$} & tiw56n58_150 & {$[1155392,1183448]$} \\
\hline tiw56n62_150 & {$[1626921,1668859]$} & tiw56n66_150 & {$[2107619,2160391]$} \\
\hline tiw56n67_150 & {$[2372926,2422085]$} & tiw56n72_150 & {$[4135907,4222250]$} \\
\hline tiw56r54_150 & {$[958192,979551]$} & tiw56r58_150 & {$[1219295,1248461]$} \\
\hline tiw56r66_150 & {$[1940755,1988549]$} & tiw56r67_150 & {$[2057645,2107042]$} \\
\hline tiw56r72_150 & {$[2823758,2888328]$} & be75eec_250 & {$[8894078,9150239]$} \\
\hline be75np_250 & {$[17819028,18473322]$} & be75oi_250 & {$[5910266,5978555]$} \\
\hline be75tot_250 & {$[30993002,32055676]$} & stabu1_250 & {$[7744014,8012535]$} \\
\hline stabu2_250 & {$[11505924,11898812]$} & stabu3_250 & {$[11909920,12298263]$} \\
\hline t59b11xx_250 & {$[8419535,8708488]$} & t59d11xx_250 & [3842563, 4015773] \\
\hline t59f $11 \mathrm{xx} \_250$ & {$[3995868,4162626]$} & t59n11xx_250 & {$[825608,854352]$} \\
\hline t65b11xx_250 & {$[17273996,17751127]$} & t65d11xx_250 & {$[9355335,9745769]$} \\
\hline t65f $11 \mathrm{xx} \_250$ & {$[8418226,8739670]$} & t65111xx_250 & {$[666664,679527]$} \\
\hline t65n11xx_250 & {$[1431605,1475116]$} & t69r11xx_250 & {$[31824632,32688871]$} \\
\hline t70b11xx_250 & {$[25411146,26133557]$} & t70d11xn_250 & {$[15212874,15833337]$} \\
\hline t70d11xx_250 & {$[16044299,16658483]$} & $\mathrm{t} 70 \mathrm{f} 11 \mathrm{xx} \_250$ & {$[13591569,14178278]$} \\
\hline t70111xx_250 & {$[1113765,1132769]$} & t70n11xx_250 & {$[2445431,2515339]$} \\
\hline t74d11xx_250 & {$[24444287,25350257]$} & t75d11xx_250 & {$[25048727,25972723]$} \\
\hline t75e11xx_250 & {$[106873878,110318580]$} & t75k11xx_250 & {$[4094877,4249417]$} \\
\hline $\mathrm{t} 75 \mathrm{n} 11 \mathrm{xx} \_250$ & {$[4528223,4658704]$} & tiw56n54_250 & {$[2099740,2182012]$} \\
\hline tiw56n58_250 & {$[2906872,3026780]$} & tiw56n62_250 & {$[4143260,4317513]$} \\
\hline tiw56n66_250 & {$[5371361,5582715]$} & tiw56n67_250 & {$[6326881,6536019]$} \\
\hline tiw56n72_250 & {$[11155793,11520848]$} & tiw56r54_250 & {$[2388249,2484343]$} \\
\hline tiw56r58_250 & {$[3060323,3185528]$} & tiw56r66_250 & {$[4948720,5147594]$} \\
\hline tiw56r67_250 & {$[5292028,5491577]$} & tiw56r72_250 & {$[7455270,7727048]$} \\
\hline
\end{tabular}

Table 15 Bounds for XLOLIB problems 